\title{
Gestão estratégica de matriculas no ensino superior: operacionalização
}

\author{
Alvaro Martins Fernades Junior* \\ Siderly do Carmo Dahle de Almeida** \\ Fernando José de Almeida***
}

\section{Resumo}

O objetivo o texto é apresentar a Gestão Estratégica de Matriculas (GEM) que pode contribuir para as discussões em captação e recrutamento de alunos no ensino superior, mas principalmente retenção. $\mathrm{O}$ texto define a GEM, e também aborda os elementos que a compõe, e finaliza expondo uma maneira das IES estruturarem ela para que possa melhorar suas ações comerciais e acadêmicas.

Palavras-chave: Gestão estratégica de matriculas. Ensino Superior. Retenção de alunos

* http://orcid.org/0000-0003-0726-1177

Centro Universitário Internacional Uninter. Doutor em Educação: Currículo pela Pontifícia Universidade Católica de São Paulo. Mestre em Gestão do Conhecimento nas Organizações pela Unicesumar. Pós-graduado em Marketing e EAD e Tecnologias Educacionais. Bacharel em Comunicação Social com ênfase em Publicidade e Propaganda (Unicesumar), licenciado em Filosofia e Pedagogia (Uninter). É professor do Centro Universitário Internacional UNINTER. alvarojunior777@gmail.com

** http://orcid.org/0000-0002-2190-7213

Centro Universitário Internacional Uninter. Doutora em Educação: Currículo pela Pontifícia Universidade Católica de São Paulo. Mestre em Educação pela Pontifícia Universidade Católica do Paraná. Bacharel em Biblioteconomia pela Universidade Federal do Paraná, licenciada em Filosofia, Pedagogia e Letras. É coordenadora do Programa de Pós-graduação stricto sensu em Educação e Novas Tecnologias do Centro Universitário Internacional Uninter. É pesquisadora da Fundação Wilson Picler de Amparo a Educação, Ciência e Tecnologia (FAMPECT). siderly.c@gmail.com

*** https://orcid.org/0000-0002-6498-3427

Pontificia Universidade Católica de São Paulo.Doutor em Filosofia da Educação pela Pontifícia Universidade Católica de São Paulo, Brasil (1984). Professor Titular do Programa de pós-graduação stricto sensu Educação: Currículo da Pontifícia Universidade Católica de São Paulo.

fernandoalmeida43@gmail.com 


\section{Strategic enrollments managent in higher educa- tion: operationalization}

\section{Abstract}

The objective of the text is to present the Strategic Enrollments Management (SEM) that can contribute to the discussions on attracting and recruiting students in higher education, but mainly retention. The text defines SEM, and also addresses the elements that compose it, and concludes by exposing a way for HEIs to structure it so that it can improve their commercial and academic actions.

Keywords: Strategic enrollment management. Higher education. Student retention

\section{Gestión estratégica de matrículas en educación superior: operacionalización}

\section{Resumen}

El objetivo del texto es presentar la Gestión Estratégica de Matrículas (GEM) que puede contribuir a las discusiones sobre la captación y captación de estudiantes en la educación superior, pero principalmente la retención. El texto define GEM, y también aborda los elementos que lo componen, y concluye exponiendo una forma para que las IES lo estructuran para que mejore sus acciones comerciales y académicas.

Palabras clave: Gestión estratégica de matrículas. Enseñanza superior. Retención de estudiantes

\section{Introdução}

Com o aumento da concorrência, as IES passam a adotar modelos de negócios mais agressivos para recrutar e matricular estudantes. $\mathrm{O}$ aumento da indústria de rankings das faculdades e a crescente importância da persistência e das taxas de graduação também resultaram em maior foco no sucesso dos alunos e nas mudanças organizacionais para apoiar esses esforços. (HOSSLER, 2015). É desta perspectiva que as IES passam a investir na melhoria de processos para recrutamentod e alunos, surgindo a Gestão Estratégica de Matrículas (GEM). 
A GEM pode ser explicada sob a ótica de duas perspectivas teóricas, a primeira é a Teoria da Dependência de Recursos, de Jeffrey Pfeffer e Gerald Salancik e a Teoria Institucional de Paul DiMaggio e Walter Powell.

Com relação a primeira, ela postula que

as organizações respondem às mudanças no ambiente externo ao mudar o tempo, a energia e os recursos para proteger ou adquirir recursos escassos que são fundamentais para a saúde e a vitalidade da organização. (HOSSLER, 2015, p. 9).

De forma simples, a teoria acredita que as organizações devem se adaptar as mudanças sempre que seu negócio estiver em risco.

Com relação a segunda, os autores afirmam que

Instituições e organizações dentro das instituições, na busca por legitimidade, muitas vezes imitam (processos miméticos) os padrões e estruturas organizacionais de outras instituições e organizações consideradas bem-sucedidas. (HOSSLER, 2015, p. 10).

Ou seja, observa-se empresas bem-sucedidas e se estuda quais processos dela podem ser incorporados, seja de maneira mimética (cópia) ou por meio de adaptações.

O conceito de gestão estratégica de matrículas(GEM) surgiu nos Estados Unidos quando estavam se debatendo, entre outros pontos, a privatização do ensino superior. E embora de origem americana, o conceito pode se aplicar a IES do mundo todo.

Inclusive no Brasil, onde as IES públicas não dão conta de atender a demanda por ensino superior, então grandes faculdades, centros universitários e universidades particulares entram "no jogo" para oferecer ensino aos que não conseguem ter acesso a educação superior gratuita. E no intuito de se diferenciarem e atrair alunos (o lucro) as IES particulares passam a fazer o uso do que se pôde aprender com outros ramos de negócios. É nesse contexto que a GEM se insere. 
Hoje em dia, o que motiva a aplicação do conceito de GEM às IES são a globalização, rankings internacionais de faculdades e universidades, e as tendências demográficas em muitas nações industrializadas. (HOSSLER, 2015).

Hossler (2015) citando Bolman e Deal (1991) define a GEM como um quadro estrutural que pode ser simultaneamente considerado como uma estrutura organizacional, como um conjunto de processos e como políticas organizacionais.

Nesta perspectiva, a GEM é simultaneamente um conjunto de processos e políticas associadas ao recrutamento e admissão de estudantes universitários, bem como a retenção, sucesso acadêmico e graduação de estudantes matriculados. Ela é responsável por toda a vida acadêmica do estudante, desde a geração de seu interesse pela IES, passando pela sua retenção enquanto aluno, até o momento que ele se forma.

Bob Bontrager e Christine Kerlin (2004) afirmam que a GEM compreende os seguintes componentes: Características da instituição e do mundo à sua volta, Missão e prioridades institucionais, Otimização das inscrições (número, qualidade, diversidade), Recrutamento de estudantes, Taxas de estudantes e ajuda financeira, Retenção, Marketing institucional, Orientação e desenvolvimento de carreira, Aconselhamento acadêmico, Desenvolvimento curricular, Métodos de ensino, Qualidade da vida e instalações do campus

Hosller e Bean (1990, p,5) colocam que a GEM consiste em uma gama de "atividades destinadas a permitir que as instituições educacionais exerçam maior influência sobre a matrícula de estudantes e a receita líquida total de mensalidades derivadas dos alunos matriculados."

A GEM é permeada por muita pesquisa acadêmica e considera como parte do processo de matrícula os seguintes pontos: a escolha do aluno por determinada IES, a transição do ensino médio para o ensino superior, atração e retenção dos alunos e, por fim, resultados estudantis. 
A GEM, indiretamente, influência na aprendizagem dos alunos dado que ela visa fornecer meios de garantir tranquilidade para que o aluno possa ingressar no ensino superior e terminar o seu curso.

A GEM ocorre em um ambiente complexo, onde as IES enfrentam sérios desafios à medida que procuram satisfazer as necessidades dos aspirantes a universitários que são cada vez mais diversificados em variáveis geográficas, étnicas, econômicas e educacionais, ao mesmo tempo em que visam alcançar os seus objetivos enquanto empresa que visa o lucro. (BONTRAGER e HOSSLER, 2015).

Importante compreender que a GEM se trata da integração de vários departamentos que costumam estar separados nas IES: aconselhamento acadêmico, ingresso de estudantes, finanças e "uma orientação para abordagem institucional abrangente destinada a permitir que os administradores da faculdade e da universidade exerçam maior influência sobre os fatores que moldam suas inscrições e matrículas". (BONTRAGER e HOSSLER, 2015)

A GEM constitui em uma orientação de marketing para o recrutamento de alunos, mas também um entendimento de que a retenção de estudantes é tão importante quanto o seu recrutamento. A GEM permite que decisões de preço de taxas podem ser usadas de forma sistemática para alcançar múltiplos objetivos de inscrição e que ela depende muito da pesquisa empírica e da análise de dados para orientar seus esforços.

Por fim, a GEM é um processo que tem implicações organizacionais e, muitas vezes, requer mudanças estruturais na forma como várias funções da universidade são integradas e organizadas em torno dos esforços para se inscrever e manter um corpo estudantil com um conjunto desejado de características.

\section{Escolhas, mercados e acesso}

No que tange as escolhas dos alunos sobre onde e o que estudar, há teorias de variadas disciplinas como economia e sociologia, e estas contribuíram muito no campo do conhecimento que 
estuda como os estudantes fazem escolhas. Porém, é sabido que na prática "a teoria não funciona assim", mas ainda sim é importante conhecer as teorias, pois elas em conjunto com a prática são uma via de mão dupla.

$\mathrm{Na}$ GEM costuma-se fazer o uso de três modelos teóricos para compreender as escolhas dos estudantes: o econômico, o sociológico e a do processamento da informação.

A abordagem de cunho econômico é sustentada por estudos ( Baum \& Payea, 2004; Perna, 2006; Leslie \& Brinkman, 1987) que afirmam que os indivíduos calculam os benefícios totais de frequentar uma faculdade (como aumento do salário, melhoria no status social, aproveitamento da experiência de aprendizagem, melhores ambientes de trabalho, melhor saúde, compras mais informadas, menor probabilidade de desemprego, etc.) e compara-os com os custos esperados (como o preço da faculdade, taxas, livros e suprimentos; oportunidades e / ou cursos alternativos, ajuda financeira, tempo de lazer, custos de viagem e assim por diante). (PARK e HOSSLER, 2015).

A abordagem sociológica comunga de alguns fatores da abordagem econômica. E da mesma maneira, afirma que a escolha por um curso é resultado da interação entre as aspirações e restrições educacionais. Ela enfatiza

as influências do capital social e cultural, como a educação dos pais, a renda familiar, a frequência que os alunos são levados para museus ou incentivados a ler pelos pais e o impacto dessas interações sociais sobre as aspirações educacionais dos estudantes (PARK e HOSSLER, 2015, p. 51)

Ao contrário da abordagem econômica que enfatiza as análises de custo-benefício nos estágios posteriores ao da escolha da faculdade, a abordagem sociológica tende a se concentrar mais nas etapas das aspirações e preparação para a faculdade.

Por fim, a abordagem de processamento da informação busca entender como os estudantes levantam, processam e tomam decisões baseadas em informações. Enquanto a abordagem econô- 
mica deslinda quais tipos de informações os estudantes usam, ela não explica de ondem os estudantes mineram as informações, foco desta última abordagem.

Há autores que estudam o processo de escolha do aluno a dividindo-o em múltiplos estágios, conforme se vê no quadro a seguir:

\begin{tabular}{|l|l|}
\hline Gregory Jackson (1982) & $\begin{array}{l}\text { com três estágios - preferência, exclusão e } \\
\text { avaliação - nos quais os alunos desenvolvem } \\
\text { sua preferência por estudar, excluem algu- } \\
\text { mas instituições com base na preferência } \\
\text { deles e desenvolvem um conjunto de con- } \\
\text { siderações e, em seguida, avaliem as carac- } \\
\text { terísticas das faculdades consideradas para } \\
\text { decidir onde estudar. }\end{array}$ \\
\hline $\begin{array}{l}\text { Katherine Hanson } \\
\text { and Larry Litten (1982) }\end{array}$ & $\begin{array}{l}\text { Com cinco estágios - nos quais os alunos } \\
\text { desenvolvem a aspiração por estudar, pes- } \\
\text { quisam informações da faculdade, reúnem } \\
\text { informações da faculdade, submetem uma } \\
\text { candidatura à faculdade e, finalmente, se } \\
\text { inscrevem em uma faculdade. }\end{array}$ \\
\hline
\end{tabular}

Fonte: Adaptado de (PARK e HOSSLER, 2015)

Essas ações de escolha acontecem dentro de um "marketplace", um mercado, uma estrutura dentro da qual os alunos comparam, consideram e escolhem entre suas opções e oportunidades de ensino superior e qual é o contexto para a manobra competitiva entre colégios e universidades em busca de seus objetivos e aspirações. (KASLBEEK e ZUCKER, 2015).

O gênero, apesar de ser considerada um importante variável preditiva não fornece muitas pistas sobre a medida que este realmente influencia. Os estudos de Hossler e Stage (1992) abordam uma grande expectativa educacional por parte das mulheres, já os estudos de Hao e Bonstead-Bruns (1998) encontraram altas expectativas por parte dos homens. Hossler e Frances Stage (1992) encontraram que as aspirações educacionais dos estudantes do sexo 
feminino aumentaram à medida que discutiam escolhas da faculdade com seus pais com mais freqüência

Park e Hossler (2015, p.53) afirmam que "a renda familiar e o status socioeconômico foram os preditores mais fortes da escolha da faculdade. A renda familiar influencia todos os resultados da escolha da faculdade, como aspirações educacionais, comportamentos de aplicação e decisões de inscrição. " Kao e Tienda (1998) viram que estudantes de baixa renda tendem a ter menor aspiração acadêmica, Avery (2010), Hill e Winston (2010) e Hurtado et al. (1997) pesquisaram que estudantes de baixa renda são menos propensos a se candidatar a faculdades de quatro anos e tendem a se motivarem menos a procurar cursos e por fim, Avery (2010), Ellwood e Kane (2000), Harper e Griffin(2011), Hossler et al. (1999), Kane e Rouse (1999) e Winston e Hill (2005) apontam em seus estudos que este perfil de estudante são menos propensos a se matricular em uma faculdade de quatro anos e são mais prováveis para se inscrever em uma faculdade de dois anos, pública e menos seletiva do que seus pares de famílias com altos rendimentos.

Capital social e cultural também são relevantes variáveis preditivas, e que nível educacional dos pais e interesses destes pela educação dos filhos enquanto estão no ensino fundamental são fatos que influenciam sobremaneira nas aspirações dos jovens ao ensino superior. (PARK e HOSSLER, 2015).

\section{Preços e ajuda financeira}

O preço é um dos 4Ps do marketing estabelecido por McCarthy, e

no composto de marketing, o preço tem natureza distinta dos demais elementos. Enquanto os outros Ps criam valor para o cliente e implicam desembolsos de dinheiro pela empresa, com o preço a empresa obtêm parte do valor propiciado como recompensa monetária, dentro da relação de troca. O preço é a contrapartida que a empresa cobra do consumidor pelo que oferece a ele (URDAN; URDAN, 2006, p. 185). 
Colocar preço é uma questão delicada, que não envolve meramente unidades monetárias, "preço é tudo que o consumidor percebe ter dado ou sacrificado para obter o produto" (URDAN; URDAN, 2006, p. 184).

O composto preço pode ser considerado ágil, dado que eles podem ser alterados de forma mais rápido do que o produto em si e seu local de distribuição, e em conjunto com o composto promoção (que vai divulgar) o novo preço, pode contribuir para atender aos anseios estratégicos de uma empresa. Porém, a sua manutenção influencia diretamente na saúde financeira da empresa.

Um aumento nas ofertas de ajuda financeira institucional aos alunos pode reduzir o preço das mensalidades e provavelmente aumentará as inscrições, mas pode afetar negativamente os orçamentos institucionais para futuras ações. As decisões de preço causam impactos nos orçamentos, e na habilidade dos tomadores de decisão para encontrar a melhor estratégia. Serna e Birnbaum (2015) argumentam da necessidade dos gestores de matrícula terem amplos conhecimentos de economia para que possam debater com superiores.

O preço da mensalidade e das taxas envolvidas na matricula estão intimamente ligadas ao lucro que IES terá, porém, pensar na percepção do público, acesso e diversidade são fatores importantes quando está se estipulando os valores que a IES cobrará por seus serviços.

Como em qualquer instituição de ensino, os seus gestores só possuem controle das variáveis internas, e em cima delas podem realizar vários cenários, mas é importante também estar ciente das variáveis externas, aquilo que afeta a empresa mas que ela não tem controle, como políticas do governo, ações da concorrência e mudança de preferência por parte dos alunos.

Independentemente do prestígio ou missão institucional, a teoria da escolha do consumidor sugere que à medida que o preço do ensino superior aumenta, indivíduos com orçamentos limitados devem fazer compensações em outras áreas de consumo ou decidir não frequentar a faculdade (PARK e HOSSLER, 2015). 
A teoria da escolha do consumidor demonstra os tradeoffs que ocorrem quando o preço de ir à faculdade aumenta sem um aumento proporcional na renda. Nesta perspectiva, os teóricos afirmam que os estudantes fazem uma escolha: ou fazem a faculdade ou fazer qualquer outra coisa que desejarem. (PARK e HOSSLER, 2015). A teoria também afirma algo que é óbvio: após pesquisar e fazer as contas, alguns estudantes se matriculam e outros não, porém, ela sugere que os gestores de matriculas invistam seus esforços nos estudantes que estão entre os extremos, cujo, uma ajuda financeira (desconto, reparcelamento) pode contribuir para a efetivação de sua matricula. (CHESLOCK; KROK, 2012).

Brooks (2015) complementa colocando que embora os descontos requeridos pelos alunos sejam totalmente atendidos, as decisões de admissão são, em menor ou menor grau, feitas dependendo do bem-estar financeiro da família. Ou seja, o preço e as negociações de descontos são variáveis relevantes, porém, a renda e reunião familiar são aspectos externos ao qual a instituição de ensino não possui controle.

\section{As operações envolvidas}

Costumeiramente, no Brasil, o departamento de matriculas e inscrições é resultado de uma força tarefa entre o departamento comercial e a secretaria acadêmica, mas aqui conheceremos a pesquisa de DeHaemers e Sandlin (2015) de como deve ser o ideal, para maior eficácia e eficiência dos processos.

Este departamento é encarregado de recrutar candidatos, processar pedidos e implementar a política de admissão da instituição, ao mesmo tempo que colabora com unidades acadêmicas em todo o campus para garantir que as metas de inscrição na universidade e os requisitos orçamentários sejam realizados. (2015, p.378).

Geralmente esse departamento trabalha em conjunto com os que realizam pesquisa, avaliação e planejamento institucional, marketing e relações públicas, bolsas de estudo, registros acadêmi- 
cos e aconselhamento acadêmico. (DEHAEMERS e SANDLIN, 2015). No Brasil, todas as instituições possuem todos esses departamentos, com exceção do último, aconselhamento acadêmico, que é privilégio de apenas algumas instituições. Um ponto óbvio, mas que deve ser salientado, é que mesmo na GEM, esses departamentos funcionam separadamente, mas com ela, se promove a melhor integração entre esses departamentos.

A estrutura organizacional no departamento de admissão deve apoiar e promover a eficiência e o serviço operacional para futuros estudantes, famílias, campus e público. (DEHAEMERS e SANDLIN, 2015). O seu trabalho se inicia ao fazer o primeiro contato com o aluno, convite para vestibular, realização do vestibular, documentação para matricula e se finda com o aluno iniciando seus estudos.

Esse departamento de admissão lida tanto com o público interno (outros departamentos da instituição) e externo (alunos e seus familiares) e por isso, é fundamental uma boa liderança e uma boa equipe em um escritório de admissão, pois o seu sucesso afeta todo o campus. (SCHUH; UPCRAFT, 2001).

DeHaemers e Sandlin (2015) abordam sobre o local onde o escritório de admissões vai se estabelecer e afirmam que suas pesquisas apontam que as instituições não costumam pensar nesse ponto de maneira estratégica, e para auxiliar os autores sugerem algumas questões a serem respondidas: É fácil de encontrar o local? Possui estacionamento? É gratuito? A sua estrutura física impressiona? O staff é amigável e conhecedor dos serviços da IES? O local possui uma sala especial para que os alunos se reúnam com conselheiros educacionais? Há algum espaço onde os estudantes podem aprofundar seus conhecimentos sobre a IES?

Para lidar com toda a complexidade envolvida, o departamento de matriculas devem ter a tecnologia como aliada, porém, mas do que é isso, é que os colaboradores sejam aliados a ela. Afinal, a instituição pode investir um bom dinheiro em softwares e hardwares, mas seus funcionários podem não saber (ou não querer) opera-los. 
Kilgore e Gage (2015) sugerem as tecnologias que podem ser utilizadas com prospects, candidatos e alunos.

\begin{tabular}{|l|l|}
\hline Prospects & E-mail, página da web, CRM e redes sociais \\
\hline Candidatos & $\begin{array}{l}\text { Aplicativos específicos para candidatos, CRM, e-mail, } \\
\text { internet, textos, portais, e redes sociais }\end{array}$ \\
\hline Aceitos & $\begin{array}{l}\text { CRM, e-mail, aplicativos, internet, textos, portais, e re- } \\
\text { des sociais }\end{array}$ \\
\hline Matriculados & $\begin{array}{l}\text { CRM, e-mail, ambientes virtuais de aprendizagem, Por- } \\
\text { tfolios de aprendizagem, aplicativos, internet, textos, } \\
\text { portais, e redes sociais }\end{array}$ \\
\hline Formados & Email, página da web, CRM e redes sociais \\
\hline
\end{tabular}

Fonte: Kilgore e Gage (2015, p. 430)

Em consonância aos sistemas tecnológicos se tem a importância da comunicação integrada, e nos bancos de dados da instituição, deveram ter informações as formais as quais os futuros alunos preferem receber interação por parte da instituição de ensino. Kilgore e Gage (2015, p.388) sugerem ainda que mensagens de texto são as mais efetivas para atrair alunos, "em um estudo de outubro de 2009 da St. Mary’s University, a instituição descobriu que os potenciais candidatos que optaram por receberem mensagens de texto eram sete vezes mais propensos a se converterem em alunos."

Uma GEM torna vários departamentos interdependentes e "cuida" desde a prospecção do aluno no mercado, até o momento em que ele se forma. A GEMs que fazem o trabalho de acompanhamento para evitar a evasão, mas isso é tópico de nossa quinta aula.

\section{Desenvolvendo um plano de gestão estratégica de matriculas}

Bontrager e Green (2015) sugerem três estruturas de funcionamento. Elas vão abordar o "quem, o como e o que" da GEM. A primeira estrutura é a das pessoas envolvidas no desenvolvimento da GEM. Na figura a seguir mais detalhes sobre essa estrutura: 


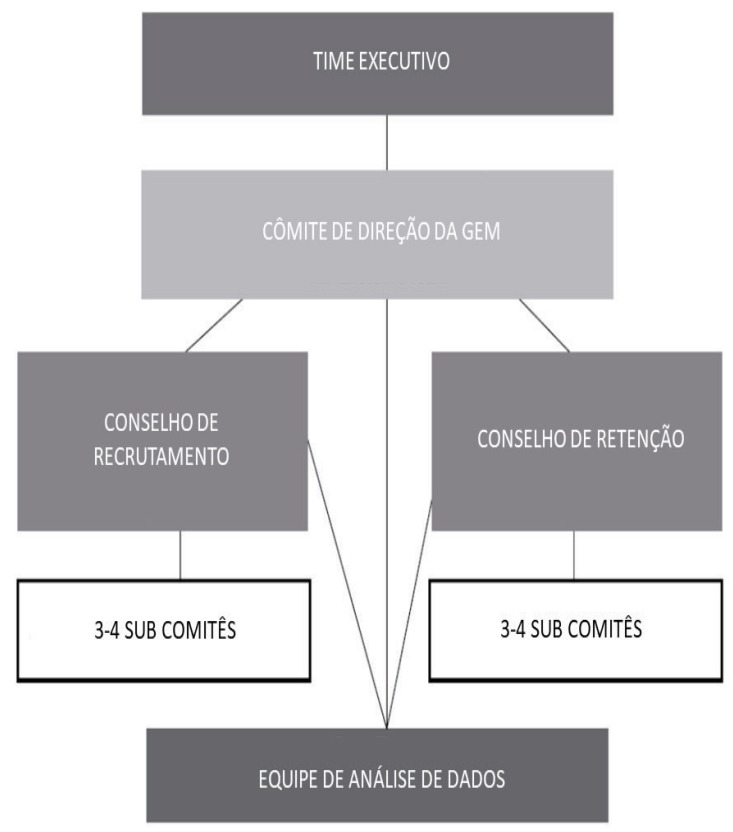

Figura - Equipe da gestão estratégica de matrículas

Fonte: BONTRAGER E GREEN (2015) (tradução dos autores)

O Time Executivo tem como função a definição do plano estratégico institucional, aprovação e defesa das metas e iniciativas estratégicas de inscrição e matriculas O Comitê de direção da GEM visa objetivos de inscrição e matriculas de longo prazo, assegurando a aprovação de estratégias através de canais institucionais apropriados e faz a comunicação com a Equipe Executiva.

O conselho de recrutamento deve desenvolver 3-4 metas estratégicas para o recrutamento de novos estudantes; revisar e aprovar os planos de ação dos seus subcomitês, e fazer recomendações ao Comitê de Direção da GEM. O conselho de retenção deve desenvolver 3-4 metas estratégicas para retenção e graduação; revisar e aprovar planos de ação dos seus subcomitês, e fazer recomendações ao Comitê de Direção da GEM. 
Todos os subcomitês (de retenção e recrutamento) devem elaborar planos de ação e cronogramas para cada meta estratégica. Por fim, a equipe de analise de dados monitoria o ambiente mercadológico, faz pesquisa de comportamento de matriculas dos estudantes, modelos de inscrição, e fornece dados aos conselhos conforme necessário. $\mathrm{O}$ desenho a seguir representa a divisão de tarefas e o fluxo de trabalho
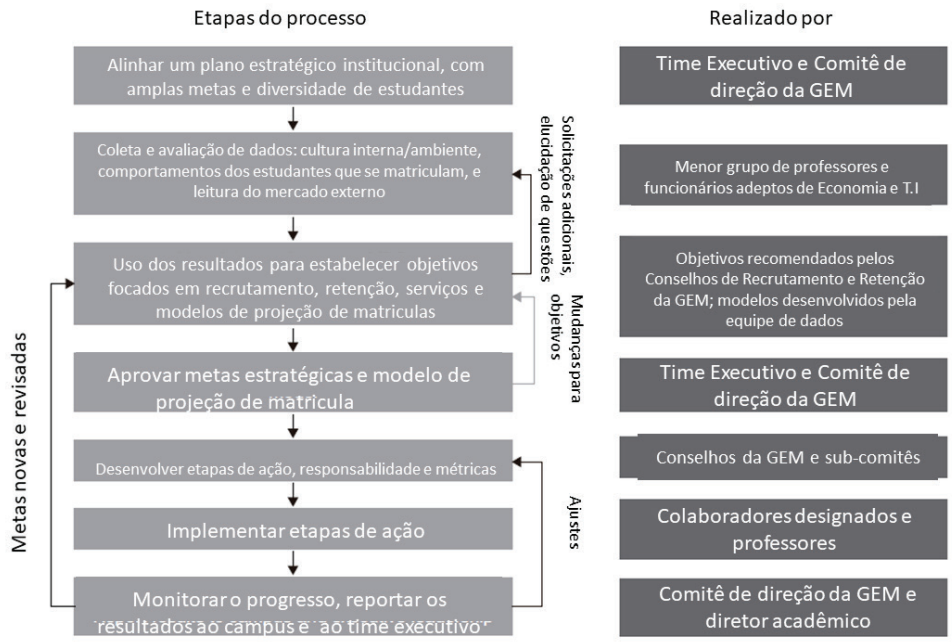

Figura - divisão de tarefas e fluxo de trabalho na GEM

Fonte: BONTRAGER E GREEN (2015) (tradução dos autores)

A GEM é oriunda de um plano de metas institucionais, definidos pelo Time Executivo e pelo Comitê de GEM. Essas metas vão sendo subdividas em ações, índices, estratégias e outras metas a serem cumpridas por todos os responsáveis por ela.

O planejamento efetivo de GEM acontece quando uma instituição funciona de baixo para cima da pirâmide, conforme veremos na figura a seguir, com os esforços de planejamento em níveis mais baixos que formam a base para os esforços acima deles. Isso fornece uma arquitetura para o desenvolvimento de planos de GEM que alcançarão resultados a longo prazo. 


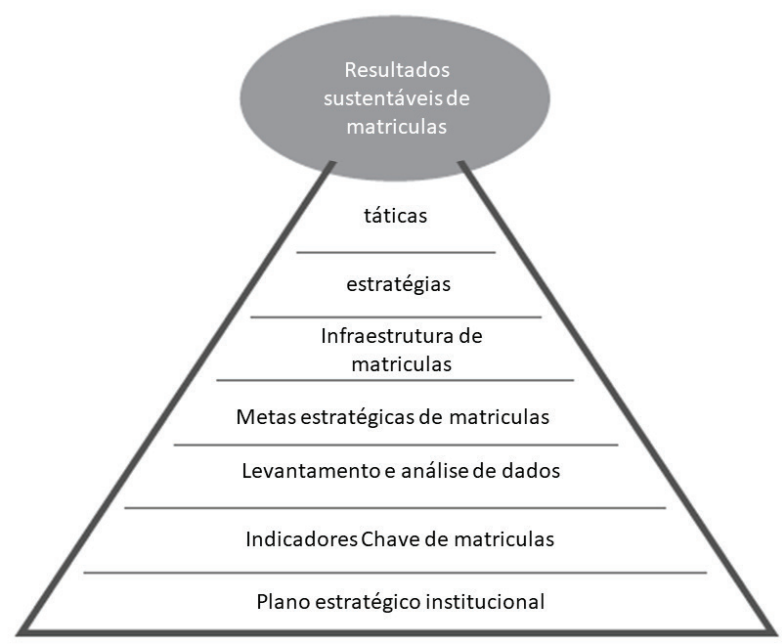

Figura - pirâmide da efetividade da GEM

Fonte: BONTRAGER E GREEN (2015) (tradução dos autores)

Com essas três estruturas sugeridas por Bontrager e Green (2015) é possível idealizar uma Gestão Estratégica de Matriculas nas IES no intuito de melhorar os índices de captação de matrículas, mas principalmente, a retenção.

\section{Considerações finais}

A GEM surgiu da necessidade das IES de adotarem modelos de negócios mais agressivos para recrutar, matricular e reter os estudantes. As provas que ranqueiam as IES também aumentaram, e isso levantou a necessidade de ter um maior foco no sucesso dos alunos, e para isso mudanças organizacionais eram necessárias.

A GEM é explicada sob a ótica da Teoria da Dependência de Recursos e da Teoria Institucional, e que a base que uma IES utiliza vai depender da sua capacidade de inovação e do seu modelo de negócios.

O texto não deixa claro se a GEM é um departamento ou um processo na IES, afinal, corroborando a literatura exposta aqui, 
os dois entendimentos são possíveis. O que realmente interessa, é que ela aconteça, seja como um processo estruturado ou como um departamento da IES.

A GEM lida com estudos sobre as escolhas dos alunos, com aconselhamento, e que costumeiramente ela "cuida" do aluno, desde o momento que ele cria interesse por estudar na instituição até o momento em que ele começa a estudar, porém, vimos também, que GEMs mais estruturadas fazem também os trabalhos de retenção de alunos.

Foi visto que ela consiste em algo estratégico que envolve diversos backgrounds de ação, e em várias fases de um processo de matricula, desde a captação até o sucesso acadêmico do aluno, sua conclusão. E por que não, após isso, para convida-lo para um processo seletivo de pós-graduação?

Foi levantada a importância de capacitação pessoal para lidar em todas as fases do processo, e também da necessidade de tecnologia de ponta principalmente a da alimentação do banco de dados e suas análises.

Por fim, foi compartilhado três estruturas sugeridas por Bontrager e Green (2015) para auxiliar as IES e os pesquisadores a compreender melhor a GEM, para que sejam aptos a estruturar processo parecidos e melhorarem a prestação de serviço educacional.

\section{Referências}

BAUM, S.; PAYEA, K. Education Pays 2004: The Benefits of Higher Education for Individuals and Society. New York: College Entrance Examination Board. 2004

BONTRAGER, B.; GREEN, T. Strategic Enrollment Planning. Em D. Hossler, \& B. Bontranger, Handbook os Strategic Enrollment Management. San Francisco, CA: Jossey-Bass. 2015.

BONTRAGER, B.; KERLIN, C. Creating Effective SEM Organizations. [PowerPoint]. Presented at American Association of Collegiate Registrars and Admissions Officers, Orlando, FL. 2004. 
BONTRAGER, B.; HOSSLER, D. Understanding the Context. Em D. Hossler, \& B. Bontrager, Handbook of Strategic Enrollment Management. San Francisco, CA: Jossey-Bass. 2015

BROOKS, S. Using Campus-Based Financial Aid Strategically. Em D. Hossler, \& B. Bontranger, Handbook os Strategic Enrollment Management. San Francisco, CA: Jossey-Bass. 2015.

CHESLOCK, J.; KROC, R. Managing college enrollments. In R. Howard, G.McLaughlin, W. Knight, \& Associates, The Handbook of Institutional Research (pp. 221-236). San Francisco: Jossey-Bass. 2012

DEHAEMERS, J.; SANDLIN, M. Delivering Effective Admissions Operations. Em D. Hossler, \& B. Bontranger, Handbook of Strategic Enrollment Management. San Francisco, CA: Jossey-Bass.2015

HAO, L.; BONSTEAD-BRUNS, M. Parent $\square$ child differences in educational expectations and the academic achievement of immigrant and native students. Sociology of Education, 71(3), 175-198. 1998.

HOSSLER, D. Origins of Strategic Enrollment Management. Em D. Hossler, \& B. Bontrager, Handbook of Strategic Enrollment Management. San Francisco, CA: Jossey-Bass. 2015.

HOSSLER, D., BEAN, J. P., et al (1990). The Strategic Management of College Enrollments. San Francisco: Jossey $\square$ Bass. 1990.

HOSSLER, D.; STAGE, F. Family and high school experience influences on the postsecondary plans of ninth grade students: A structural model of predisposition to college. American Educational Research Journal, 29(2), 425-451. 1992.

KASLBEEK, D. H.; ZUCKER, B. Markets and Market Niches. Em D. Hossler, \& B. Bontrager, Handbook of Strategic Enrollment Management. San Francisco, CA: Jossey-Bass. 2015.

KILGORE, W., \& GAGE, B. The Role of Technology in Supporting SEM. Em D. Hossler, \& B. Bontrager, The Handbook os Strategic Enrollment Management. San Francisco, CA: Jossey-Bass. 2015.

LESLIE, L. L.; BRINKMAN, P. T. Institutional factors and access student price response in higher education: The student demand studies. The Journal of Higher Education, 58(2), 181-204. 1987. 
PARK, E.; HOSSLER, D.. Understanding Student Choice. Em D. Hossler, \& B. Bontranger, Handbook of Strategic Enrollment Management. San Francisco, CA: Jossey-Bass. 2015.

PERNA, L. W. Studying college access and choice: A proposed conceptual model. In J. C. Smart (Ed.), Higher Education: Handbook of Theory and Research, Vol. 21, (pp. 99-157). The Netherlands: Springer. 2006.

SERNA, G. R.; BIRNBAUM, M. Economic Perspectives on Pricing and What It Means for SEM. Em D. Hossler, \& B. Bontranger, Handbook of Strategic Enrollment Management. San Francisco, CA: Jossey-Bass. 2015.

SCHUH, J.; UPCRAFT, L. Assessment Practice in Student Affairs: An Applications Manual. San Francisco: Jossey $\square$ Bass. 2001.

URDAN, F. T.; URDAN, A. T. Gestão do Composto de Marketing. São Paulo: Atlas, 2006.

Submetido em: 22-12-2020

Aceito em: 27-7-2021 